\title{
Belief propagation-based joint iterative algorithm for detection and decoding in asynchronous CDMA satellite systems
}

\author{
$\mathrm{Na} \mathrm{Gu}{ }^{1 *}$, Sheng $\mathrm{Wu}^{1}$, Linling Kuang ${ }^{2}$, Zuyao $\mathrm{Ni}^{3}$ and Jianhua $\mathrm{Lu}^{1}$
}

\begin{abstract}
As the geostationary orbit (GEO) is congested in the commercial Ku band, the adjacent satellites will receive the interference from small aperture terminals which have a wide field of view. To reduce the interference toward the adjacent satellites, code division multiple access (CDMA) with spread spectrum needs to be adopted in the satellite communications on-the-move system. However, the mutual interference between CDMA users leads to the performance loss, which is not acceptable for the power-sensitive small aperture terminals, and the computation complexity is still an intractable problem when the conventional iterative minimum mean square error (MMSE) scheme is employed to suppress the multiple access interference (MAI) in satellite systems especially that is coded by low-density parity-check (LDPC) code. Moreover, the long transmission delays of different users caused by the long transmission distance require the robustness of the interference suppression for the asynchronous case. In this paper, a joint chip-level algorithm for CDMA multiuser detection and LDPC decoding with a new and simple iterative structure based on belief propagation (BP) for satellite systems is developed, which integrates the iterations of multiuser detection and the iterations of LDPC decoding with factor graphs. Through analysis and simulations, it is verified that the proposed algorithm is robust to the asynchronous CDMA satellite system, and with the similar iterative complexity, the performance of the proposed algorithm outperforms that of the conventional iterative MMSE scheme in the asynchronous CDMA satellite system.
\end{abstract}

\section{Introduction}

Currently, the geostationary orbit (GEO) is extremely congested in the commercial Ku band with only a $2^{\circ}$ spacing between the satellites. Small aperture terminals have a wide field of view and consequently will transmit a significant amount of power toward adjacent satellites, causing the interference at these satellites to increase, which is a main constraint for the development of the satellite communications on-the-move system. In order to limit the interference to neighboring satellites, International Telecommunication Union (ITU) has imposed strict limits on the off-axis emissions from the satellite terminal [1]. As the current satellite communications on-the-move systems are always developed on the higher frequency bands, e.g., $\mathrm{Ku}$ and $\mathrm{Ka}$ bands, which also provide larger

\footnotetext{
*Correspondence: gun08@mails.tsinghua.edu.cn

${ }^{1}$ Department of Electronic Engineering, Tsinghua University, Beijing 100084,

China

Full list of author information is available at the end of the article
}

bandwidth. Code division multiple access (CDMA) with spread spectrum is a necessary choice to reduce the interference toward the adjacent satellites. However, the multiuser CDMA system is always interference-limited, and the transmit power of the terminal needs to be increased for higher performance, which leads to the increase of the interference. This is also not acceptable for the power-sensitive small aperture terminals. Moreover, the transmission distance is always very long in satellite systems, which causes different and long transmission delays of different CDMA users. In the satellite communication system, the maximum transmission delay may be several milliseconds. Just as the assumption in [2], the inter-symbol interference (ISI) is always not considered in the CDMA satellite system, in which the power of the main path is much higher than that of the multipath components. Therefore, the satellite communications onthe-move system with CDMA can be seen as a typical asynchronous CDMA system with the transmission delays

\section{是 Springer}

(c) 2013 Gu et al: licensee Springer. This is an Open Access article distributed under the terms of the Creative Commons

Attribution License (http://creativecommons.org/licenses/by/2.0), which permits unrestricted use, distribution, and reproduction in any medium, provided the original work is properly cited. 
which are the integer multiples of chip, in which the orthogonality of different CDMA users' spreading codes is destroyed and the robustness of the interference suppression for the asynchronous case is necessary.

In order to suppress the multiple access interference (MAI) in CDMA systems, multiuser detection (MUD) needs to be adopted. An important class of the practical multiuser detectors is based on linear detection, for instance, decorrelator (a.k.a. zero-forcing equalizer), linear minimum mean square error (MMSE) detector, and so on [3,4]. In [5-7], an iterative MMSE detection algorithm for the coded CDMA system was presented, which feeds the extrinsic information of the decoder back to the iterative MMSE detector. Thus, a turbo processing framework for iterative detection and decoding was derived. In recent years, a more in-depth review about turbo equalization was given in [8]. However, when iterative decoding is adopted, the total latency of the decoder and detection increases with the iterative times of the detection, which means more computation complexity and more processing time, increasing the difficulty of engineering implementation. The belief propagation (BP)-based algorithms [9-11] are known as another class of promising approaches to detection, which were recently used in MIMO and ISI channels [12,13]. For direct-spread CDMA systems, Kabashima [14] has introduced a tractable BPbased multiuser detection algorithm, which can be operated in a practical time scale by utilizing the central limit theorem. In [15] and [16], Montanari and Tse analyzed the performance of the BP-based CDMA detection and proved that the multiuser detection algorithm based on BP is asymptotically optimal for sparse CDMA systems with Gaussian noise. An extended analysis in [17] shows that the optimality of BP-based detection in the largesystem limit is a unique advantage of sparsely spread CDMA systems, which is in contrast to the wisdom for low-density parity-check (LDPC) codes and the success of iterative decoding techniques. Also, some theoretical conclusions about the CDMA channel are obtained. However, in these studies, the BP-based CDMA iterative detection and the iterative decoding are recognized as two individual modules. Even for the joint processing, they are jointly processed only through the extrinsic information from the decoder just as the conventional joint detection and decoding structure, e.g, the iterative MMSE scheme [8] which is verified to be optimal. The iterative complexity of this conventional joint structure is still an intractable problem. Moreover, in the conventional iterative MMSE scheme, only the extrinsic information from the decoder without that from the previous iteration of the detection is employed to update the probabilistic information for detection, which induces the performance loss of joint detection and decoding.
In this paper, a joint chip-level iterative algorithm for the asynchronous LDPC-coded CDMA satellite system is proposed. Currently, LDPC codes are widely adopted $[18,19]$ in middle-rate (e.g., $1 \mathrm{Mbps}$ ) satellite communications on-the-move systems. LDPC code can easily be cast into factor graphs, and these factor graphs turn out to have cycles, leading to iterative decoding algorithms. In order to utilize the iterative structure of the LDPC decoder, we construct a global factor graph with only a global iteration, which naturally integrates the iterations of LDPC decoding and the iterations of BP-based detection.

The internal iterations of the LDPC decoder are proposed to be incorporated into the global iterations of the BP-based detector, which means the iterations of decoding are performed along with the iterative detection rather than independently performed. The modification on the iterative structure effectively simplifies the iterative structure and reduces the total iterative complexity, which is the most significant difference compared with the joint structure in [8] and [17]. By appropriately introducing the central limit theorem, the computation complexity of the derived algorithm is proportional to the square of the number of users. Furthermore, the messages of the previous detection iteration from the other users are also adopted in the message updating based on BP during every global iteration, which are more accurate about the semantics of the factor graph compared with that in [8], leading to the performance gain. It is verified that the proposed algorithm can achieve an attractive performance compared with the conventional joint detection and decoding scheme based on turbo iterative structure [8]. Practically, the satellite CDMA systems are always asynchronous due to the long transmission delays. Therefore, in this paper, through analysis and simulation, it is verified that the proposed algorithm is also robust to the asynchronous CDMA satellite system.

The rest of this paper is structured as follows: In Section 2, a system model for the asynchronous satellite CDMA system is formulated. In Section 3, the joint iterative algorithm for CDMA multiuser detection and LDPC decoding is investigated. In Section 4, the bit error rate (BER) performance is evaluated through numerical simulations and compared with that of the conventional iterative MMSE algorithm [8]. Finally, conclusions are given in Section 5.

\subsection{Notations}

Lowercase letters (e.g., $x$ ) denote scalars, bold lowercase letters (e.g., $\mathbf{x}$ ) denote column vectors, and bold uppercase letters (e.g., $\mathbf{X}$ ) denote matrices. The superscript $T$ and $\dagger$ denote the transpose operation and conjugate transpose operation, respectively. The symbol I denotes an identity matrix. 


\section{System description}

We focus on the reverse link of the asynchronous CDMA satellite system, which is an LDPC-coded directsequence binary phase-shift keying (DS/BPSK) CDMA system with $K$ users. Each user's signal is spreaded by random binary spreading sequences or gold sequences with different transmission delays. The spreading factor is $N$. The transmitted signal of the $k$ th user at the chip rate in a baseband discrete time model representation is

$$
u_{k}[n]=\sum_{l=-\infty}^{\infty} \tilde{x}_{k}[l] g_{k}\left[n-d_{k}-l N\right]
$$

where subscript $k$ denotes the user index, $g_{k}[n]$ represents the effective signature sequence of user $k$, and $0 \leq d_{k}<N$ is the transmission delay $(\bmod N)$ of user $k$ in chip periods. $\left\{\tilde{x}_{k}[l]\right\}$ is the BPSK mapped symbols of the $k$ th user from a finite set of alphabets $\{ \pm 1\}$, which is obtained by mapping the interleaved LDPC-encoded symbols $\left\{\tilde{c}_{k}[l]\right\}$ to BPSK symbols. As is shown in Figure 1, the input signal of LDPC encoder, which is called the information bits, is marked as $\left\{b_{k}[t] ; t=1,2, \ldots, T\right\}$, where $T$ is the number of information bits per user per frame. Also, the output of the LDPC encoder, which is called the coded symbols, is marked as $\left\{c_{k}[l] ; l=1,2, \ldots, L\right\}$, where $L$ is the number of symbols per user per frame after LDPC is encoded.

Assume that $\left\{s_{k}[1], s_{k}[2], \ldots, s_{k}\left[L_{c}\right] ; s_{k}[m]= \pm 1\right\}$ is the preassigned spreading code of the $k$ th user; $L_{c}$ is the code length. As the spreading factor is $N$, there are $N$ chips per symbol. The signature waveform of the $k$ th user is defined as

$$
g_{k}[n]=\sum_{m=0}^{N-1} s_{k}[m] h_{k}[n-m],
$$

where $\left\{h_{k}[n]\right\}$ denotes the effective channel impulse response (IR) between the users and the receiver sampled at the chip interval.

As the uplink channel for the satellite communications on-the-move system is usually described as an additive white Gaussian noise (AWGN) model [20], we consider an asynchronous CDMA baseband signal from $K$ users with an additive white Gaussian noise. In this paper, firstly, we assume that the signal power of each user is completely controlled at equal energy and then we extend the simulation to distributed power constraints. In the perfect power control case, the received signal is

$$
\begin{aligned}
& y[n]=\sum_{k=1}^{K} u_{k}[n]+\omega[n] \\
& =\sum_{k=1}^{K} \sum_{l=-\infty}^{\infty} \tilde{x}_{k}[l] g_{k}\left[n-d_{k}-l N\right]+\omega[n],
\end{aligned}
$$

where $\omega[n]$ is an additive Gaussian noise with zero mean and the variance $\sigma^{2}$.

By now, the received signal $y[n]$ in the asynchronous case is given, which is a mixture of different users with different transmission delays. In fact, the synchronous CDMA system can be seen as a special case with the transmission delays $d_{k}=0,(k=1,2, \ldots, K)$. In the receiver of the asynchronous case, a timing and synchronization

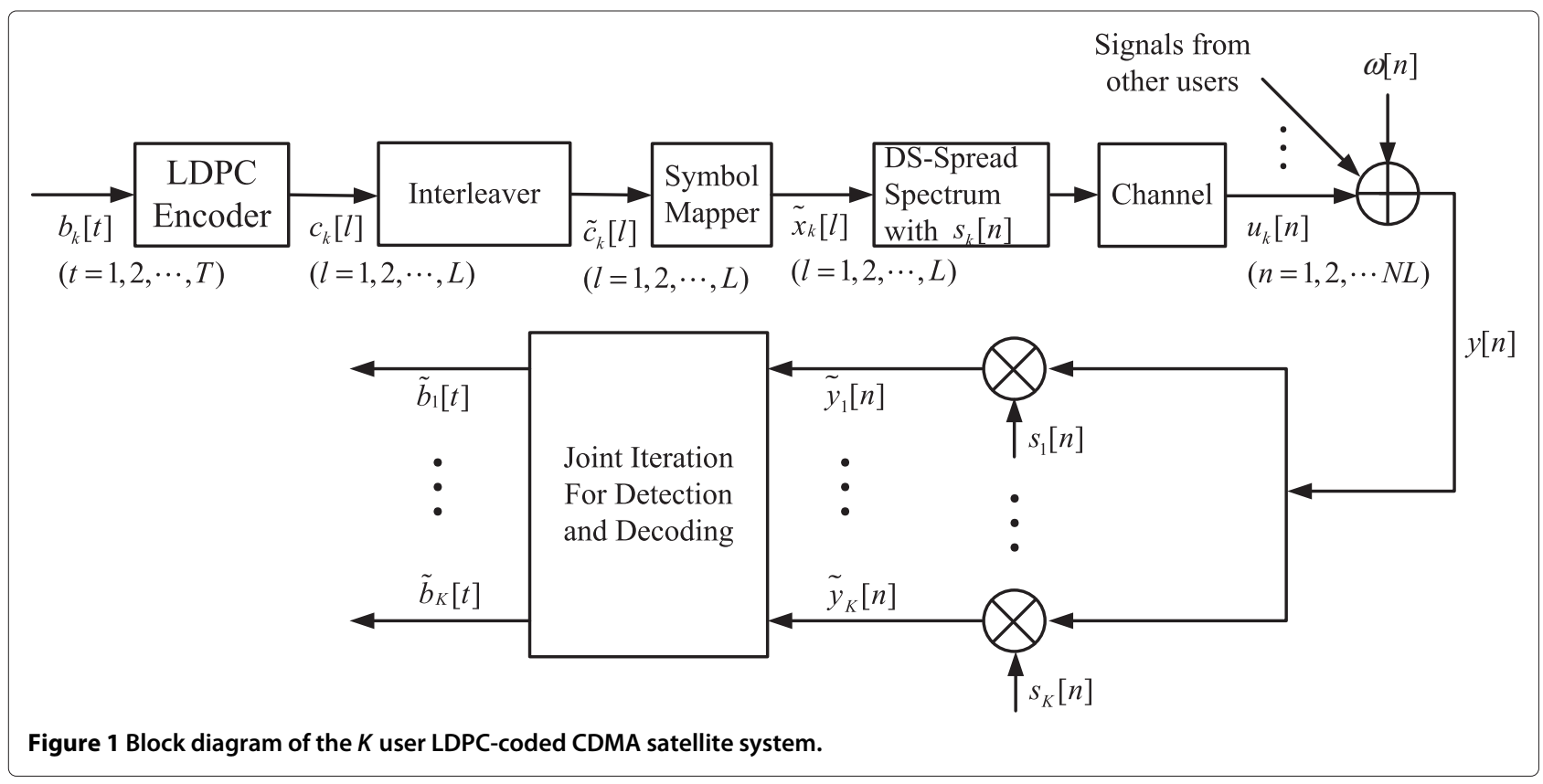


module can be applied to obtain the corresponding propagation delay $d_{k}$ of the $k$ th user, which can only be used for the alignment of the user $k$ 's spreading sequence. However, the interferences from the other users are still not aligned with the user $k$ 's signal, and the orthogonality of different users' spreading sequences is destroyed. Therefore, the asynchronization between different users is always established. In order to investigate the robustness of the proposed algorithm in the asynchronous satellite CDMA system, the asynchronous CDMA system model is applied throughout the article.

\section{Joint iterative algorithm for detection and decoding}

In this section, we present the joint chip-level iterative algorithm for CDMA multiuser detection and LDPC decoding. Firstly, the joint probability distribution of the information bits from all users is represented as the factor graph. The messages passed in the joint iterative algorithm are derived. Then, the performance advantage of the proposed algorithm is discussed. Finally, the iterative complexity of the proposed algorithm is compared with that of the conventional MMSE algorithm [8], which is also referred to as the iterative MMSE algorithm in the following discussion.

\subsection{The factor graph representation of the CDMA system}

To introduce the algorithm to CDMA systems, we graphically express the received symbols, transmitted chips, coded symbols and information bits by several kinds of nodes, and connect them by edges when they are related. The joint probability distribution of the received symbols, transmitted chips, coded symbols and information bits from all the $K$ users may be expressed as

$$
\begin{aligned}
& p(\tilde{\mathbf{Y}}, \mathbf{b}, \mathbf{c}, \tilde{\mathbf{x}}, \mathbf{X} \mid \tilde{\mathbf{H}}) \\
= & p(\mathbf{b}) p(\tilde{\mathbf{Y}}, \mathbf{c}, \tilde{\mathbf{x}}, \mathbf{X} \mid \mathbf{b}, \tilde{\mathbf{H}}) \\
= & p(\mathbf{b}) p(\mathbf{c} \mid \mathbf{b}) p(\tilde{\mathbf{x}} \mid \mathbf{c}) p(\mathbf{x} \mid \tilde{\mathbf{x}}) p(\tilde{\mathbf{Y}} \mid \mathbf{X}, \tilde{\mathbf{H}}) \\
\propto & \prod_{1 \leq i \leq K} p\left(c_{i}[l] \mid b_{i}[t]\right) \prod_{1 \leq i \leq K} p\left(\tilde{x}_{i}[l] \mid c_{i}[l]\right) \\
& \times \prod_{1 \leq i \leq K} p\left(x_{i}[n] \mid \tilde{x}_{i}[l]\right) \prod_{1 \leq n \leq N L} p(\tilde{\mathbf{y}}[n] \mid \mathbf{x}[n], \tilde{\mathbf{H}}[n]),
\end{aligned}
$$

where $\tilde{\mathbf{Y}} \in \mathcal{R}^{K \times N}$ represents the received signal during the $N$ chip intervals after multiplied by each user's spreading sequence at the satellite, and at the single chip interval, the columns of it can be expressed as

$$
\tilde{\mathbf{y}}[n]=\left[\tilde{y}_{1}[n], \tilde{y}_{2}[n], \ldots, \tilde{y}_{K}[n]\right]^{\mathrm{T}}(n=1,2, \ldots, N L) ;
$$

b denotes the vector of the corresponding information bits $\left\{b_{k}[t]\right\}(k=1,2, \ldots, K)$, $\mathbf{c}$ denotes the vector of the corresponding coded symbols $\left\{c_{k}[l]\right\}(k=1,2, \ldots, K)$, and $\tilde{\mathbf{x}}$ denotes the vector of the mapped symbols before spreading $\left\{\tilde{\mathbf{x}}_{k}[l]\right\}(k=1,2, \ldots, K) . \mathbf{X} \in \mathcal{R}^{K \times N}$ is obtained by only spreading the mapped symbols at the $l$ th interval $\tilde{\mathbf{x}}_{k}[l]$ by the spreading factor $N$ respectively without the effect of multiplying the spreading sequences in the transmitter. $\mathbf{x}[n]$ denotes the $n$th column vector of it, which is expressed as

$$
\mathbf{x}[n]=\left[x_{1}[n], x_{2}[n], \ldots, x_{K}[n]\right]^{\mathrm{T}}(n=1,2, \ldots, N L) .
$$

According to the above factorization, a factor graph for CDMA system with $K=2$, for simplification, is shown in Figure 2. The four kinds of nodes are discerned in the factor graph as follow.

1. The nodes on the top represent $p\left(c_{i}[l] \mid b_{i}[t]\right)$, $i=1,2, \ldots, K$, the relation between the information bits and coded symbols transmitted from each user.

2. The nodes in the middle represent $p\left(\tilde{x}_{i}[l] \mid c_{i}[l]\right)$, $i=1,2, \ldots, K$, the relation between the coded symbols and the mapped symbols transmitted from each user before spreading. We will perform the BP algorithm on these nodes to de-map the mapped symbols to the coded symbols or map the coded symbols to the mapped symbols.

3. The bottom-second nodes represent $p\left(x_{i}[n] \mid \tilde{x}_{i}[l]\right)$, $i=1,2, \ldots, K$, the relation between the mapped symbols before spreading and chips after spreading. We will perform the product of every $N$ chips' likelihood probability on this node to complete the accumulation of the despreading after multiplied by each user's spreading sequence, where $N$ is the spreading factor.

4. Finally, the bottom-most nodes represent the function $p(\tilde{\mathbf{Y}} \mid \mathbf{X}, \tilde{\mathbf{H}})$, the relation between the transmitted chips and the received symbols after multiplied by each user's spreading sequences. Since the satellite channel is assumed memoryless,

$$
\begin{aligned}
& p(\tilde{\mathbf{Y}} \mid \mathbf{X}, \tilde{\mathbf{H}}) \text { is factored as } \\
& \prod_{1 \leq n \leq N L} p(\tilde{\mathbf{y}}[n] \mid \mathbf{x}[n], \tilde{\mathbf{H}}[n]) .
\end{aligned}
$$

More specially, the variable nodes labeled with ' $=$ ' represent the constraint of equality. The observation nodes labeled with $\tilde{y}_{i}[n]$ represent the observation function $g_{i, n}(\mathbf{x}[n])$, which is the transition probability between $\tilde{y}_{i}[n]$ and $\mathbf{x}[n]$ only including the effect of multiplying each user's spreading sequence in the receiver without cumulation. In the proposed algorithnm, the despreading is performed in two steps: the multiplication and 


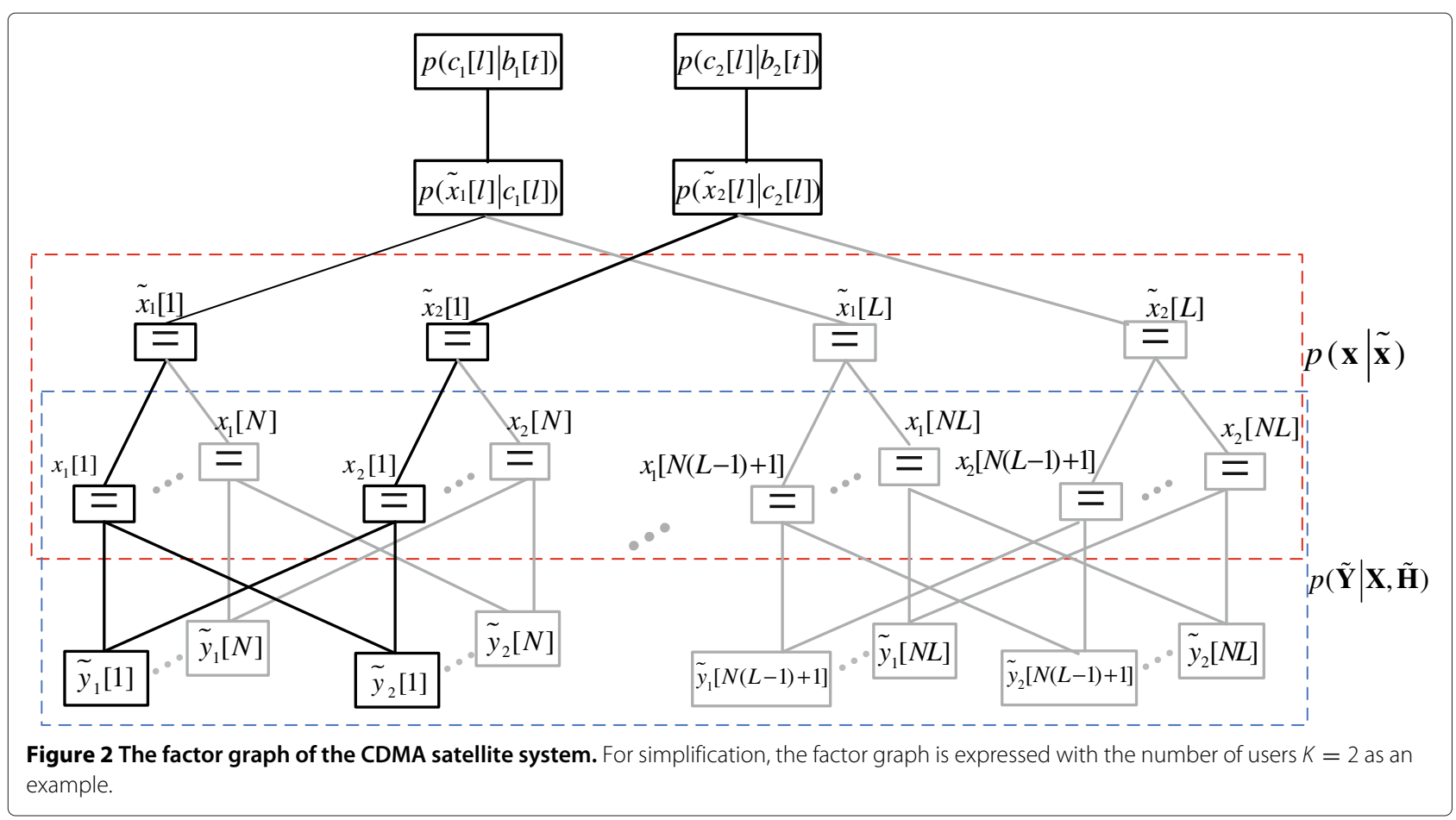

accumulation, instead of the correlation calculation. The cumulation is done to complete the despreading during updating the message from $x_{i}$ to $\tilde{x}_{i}$. Here, the transition probability function is expressed as

$$
\begin{aligned}
g_{i, n}(\mathbf{x}[n]) & =p\left(\tilde{y}_{i}[n] \mid x_{1}[n], \ldots, x_{K}[n]\right) \\
& \propto \exp \left\{-\frac{\left|\tilde{y}_{i}[n]-\sum_{j=1}^{K} \tilde{h}_{i, j}[n] x_{j}[n]\right|^{2}}{\sigma^{2}}\right\},
\end{aligned}
$$

where $\tilde{h}_{i, j}[n]$ is the element of the equivalent channel matrix between $\tilde{y}_{i}[n]$ and $x_{j}[n]$ which incorporates the effect of multiplying the spreading sequences $s_{i}\left[n-d_{i}\right]$ in the receiver with the effective signature sequences from the transmitter for the asynchronous case without the sum over $n$, expressed as

$$
\tilde{h}_{i, j}[n]=h_{j}[n] s_{j}\left[n-d_{j}\right] s_{i}\left[n-d_{i}\right],(n=1,2, \ldots, N L),
$$

where $N$ is the spreading factor and $h_{j}[n]$ represents the effective channel IR of the $j$ th user. As the satellite channel is always stable, slowly time-varying, and non-multipath, it is always easy to be obtained in the receiver. Therefore, it is assumed to be known in the receiver just as the assumption in [2]. $s_{i}[n]$ and $s_{j}[n]$ represent the respective spreading sequences for the $i$ th and $j$ th users, and $d_{i}$ and $d_{j}$ denote the transmission delay $(\bmod N)$ of the user $i$ and the user $j$, respectively.

\subsection{Message passing of the joint iterative algorithm on the factor graph}

$\mathrm{BP}$ algorithm [21,22] was widely applied to the demapper/mapper nodes and decoder nodes of the factor graph which have been described well in many literatures $[10,23,24]$. To compute $p(\tilde{\mathbf{y}}[n] \mid \mathbf{x}[n], \tilde{\mathbf{H}}[n]), 1 \leq n \leq N L$, which is always called multiuser detection, the application of the BP algorithm at the bottom-most nodes is necessary. Let $\mu_{x_{i}[n] \rightarrow \tilde{y}_{j}[n]}^{t}\left(x_{i}[n]\right)$ denote the message sent from the variable node $x_{i}[n]$ to the observation node $\tilde{y}_{j}[n]$ at the $t$ th iteration and $\mu_{\tilde{y}_{j}[n] \rightarrow x_{i}[n]}^{t}\left(x_{i}[n]\right)$ denote the message in the opposite direction. Let $\mu_{\tilde{x}_{i}[l] \rightarrow x_{i}[n]}^{t}$ denote the message sent from the node before spreading to that after spreading at the $t$ th iteration and $\mu_{x_{i}[l] \rightarrow \tilde{x}_{i}[n]}^{t}$ denote the message in the opposite direction. $\mathcal{N}(v)$ denotes the set of neighbors of a given node $v$. Thus, the message computations performed at variable and observation nodes are, respectively,

$$
\begin{aligned}
\mu_{x_{i}[n] \rightarrow \tilde{y}_{j}[n]}^{t}\left(x_{i}[n]\right)= & \mu_{\tilde{x}_{i}[l] \rightarrow x_{i}[n]}^{t}\left(x_{i}[n]\right) \prod_{p \in \mathcal{N}\left(x_{i}[n]\right) \backslash} \\
& \times \mu_{\tilde{y}_{p}[n] \rightarrow x_{i}[n]}^{t-1}\left(x_{i}[n]\right) \\
\mu_{\tilde{y}_{j}[n] \rightarrow x_{i}[n]}^{t}\left(x_{i}[n]\right)= & \sum_{\sim\left\{x_{i}[n]\right\}} g_{j, n}(\mathbf{x}[n]) \prod_{p \in \mathcal{N}\left(\tilde{y}_{j}[n]\right) \backslash i} \\
& \times \mu_{x_{p}[n] \rightarrow \tilde{y}_{j}[n]}^{t-1}\left(x_{p}[n]\right),
\end{aligned}
$$


where $\mu_{\tilde{x}_{i}[l] \rightarrow x_{i}[n]}^{t}\left(x_{i}[n]\right)$ is defined by the extrinsic probability mass function (pmf) $\mu_{\mathrm{dec} \rightarrow \tilde{x}_{i}[l]}^{t}\left(\tilde{x}_{i}[l]\right)$ fed from the decoder.

The extrinsic information from the decoder $\mu_{\mathrm{dec} \rightarrow \tilde{x}_{i}[l]}^{t}\left(\tilde{x}_{i}[l]\right)$ and the messages obtained at the previous iteration $\mu_{\tilde{y}_{j}[n] \rightarrow x_{i}[n]}^{t-1}\left(x_{i}[n]\right)$ are involved in the computation (9). This is different from the conventional iterative MMSE algorithm [8], in which only the extrinsic information from the decoder is involved. Obviously, the proposed algorithm based on the semantics of the factor graph is more accurate than the conventional algorithm, so the performance of the proposed algorithm should outperform that of the conventional iterative MMSE algorithm, which will be shown by the following simulation.

The application of the BP algorithm in the operation (10) leads to computational explosion so that the direct usage of the proposed algorithm is infeasible for CDMA system. In order to approximately carry out the belief updating in a practical time scale, the appropriate application of the central limit theorem is a classic simplification [14]. First, by minimizing the Kullback-Leibler (KL) divergence $\operatorname{KL}\left(\mu_{x_{i}[n] \rightarrow \tilde{y}_{j}[n]}^{t}\left(x_{i}[n]\right) \| \hat{\mu}_{x_{i}[n] \rightarrow \tilde{y}_{j}[n]}^{t}\left(x_{i}[n]\right)\right)$, the non-Gaussian PDF $\mu_{x_{i}[n] \rightarrow \tilde{y}_{j}[n]}^{t}\left(x_{i}[n]\right)$ can be replaced with the Gaussian PDF $\hat{\mu}_{x_{i}[n] \rightarrow \tilde{y}_{j}[n]}^{t}\left(x_{i}[n]\right)$, given as follows [25]:

$$
\begin{aligned}
& \mu_{x_{i}[n] \rightarrow \tilde{y}_{j}[n]}^{t}\left(x_{i}[n]\right) \cong \hat{\mu}_{x_{i}[n] \rightarrow \tilde{y}_{j}[n]}^{t}\left(x_{i}[n]\right) \\
& =\mathcal{N}\left(x_{i}[n], \hat{m}_{x_{i}[n] \rightarrow \tilde{y}_{j}[n]}^{t}, \hat{v}_{x_{i}[n] \rightarrow \tilde{y}_{j}[n]}^{t}\right),
\end{aligned}
$$

where

$$
\begin{gathered}
\hat{m}_{x_{i}[n] \rightarrow \tilde{y}_{j}[n]}^{t}=\sum_{\alpha_{i} \in\{-1,1\}} \alpha_{i} \mu_{x_{i}[n] \rightarrow \tilde{y}_{j}[n]}^{t}\left(x_{i}[n]=\alpha_{i}\right), \quad \\
\hat{v}_{x_{i}[n] \rightarrow \tilde{y}_{j}[n]}^{t}=\sum_{\alpha_{i} \in\{-1,1\}}\left|\alpha_{i}-\hat{m}_{x_{i}[n] \rightarrow \tilde{y}_{j}[n]}^{t}\right|^{2} \mu_{x_{i}[n] \rightarrow \tilde{y}_{j}[n]}^{t}\left(x_{i}[n]=\alpha_{i}\right) .
\end{gathered}
$$

Then, the computation of (10) can be approximate to some simple linear operations.

$$
\begin{aligned}
& \mu_{\tilde{y}_{j}[n] \rightarrow x_{i}[n]}^{t}\left(x_{i}[n]\right)= \sum_{\sim\left\{x_{i}[n]\right\}} g_{j, n}(\mathbf{x}[n]) \prod_{p \in \mathcal{N}\left(\tilde{y}_{j}[n]\right) \backslash i} \\
& \times \mu_{x_{p}[n] \rightarrow \tilde{y}_{j}[n]}^{t-1}\left(x_{p}[n]\right) \\
& \cong \int_{\sim\left\{x_{i}[n]\right\}} g_{j, n}(\mathbf{x}[n]) \prod_{p \neq i} \mathcal{N}\left(x_{p}[n], \hat{m}_{x_{p}[n] \rightarrow \tilde{y}_{j}[n]}^{t}, \hat{v}_{x_{p}[n] \rightarrow \tilde{y}_{j}[n]}^{t}\right) \\
& \propto \mathcal{N}\left(\tilde{h}_{j, i}[n] x_{i}[n], \operatorname{mean}_{\tilde{y}_{j}[n] \rightarrow x_{i}[n]}^{t}, \operatorname{var}_{\tilde{y}_{j}[n] \rightarrow x_{i}[n]}^{t}\right),
\end{aligned}
$$

where

$$
\begin{gathered}
\operatorname{mean}_{\tilde{y}_{j}[n] \rightarrow x_{i}[n]}^{t}=\tilde{y}_{j}[n]-\sum_{p \neq i} \tilde{h}_{j, p}[n] \hat{m}_{x_{p}[n] \rightarrow \tilde{y}_{j}[n]}^{t}, \\
\operatorname{var}_{\tilde{y}_{j}[n] \rightarrow x_{i}[n]}^{t}=\sum_{p \neq i} \tilde{h}_{j, p}[n] \hat{v}_{x_{p}[n] \rightarrow \tilde{y}_{j}[n]}^{t} \tilde{h}_{j, p}^{*}[n]+\sigma^{2} .
\end{gathered}
$$

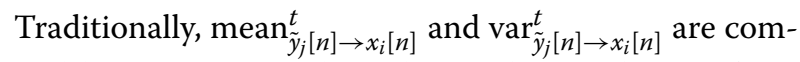
puted by summation; thus, the results are distinct from one another. For the simplified implementation, we can take advantage of the correlation between the different messages transmitted from a certain node. Instead of computing each message in a certain node separately, each message can be obtained from a sum by means of a subtraction or an addition corresponding to each node:

$$
\begin{aligned}
& \operatorname{mean}_{\tilde{y}_{j}[n] \rightarrow x_{i}[n]}^{t}=\tilde{y}_{j}[n]-\sum_{p \neq i} \tilde{h}_{j, p}[n] \hat{m}_{x_{p}[n] \rightarrow \tilde{y}_{j}[n]}^{t} \\
& =\operatorname{mean}_{\tilde{y}_{j}[n]}^{t}+\tilde{h}_{j, i}[n] \hat{m}_{x_{i}[k] \rightarrow \tilde{y}_{j}[n]}^{t}
\end{aligned}
$$

$$
=\operatorname{var}_{\tilde{y}_{j}[n]}^{t}-\left|\tilde{h}_{j, i}\right|^{2} \hat{v}_{x_{i}[n] \rightarrow \tilde{y}_{j}[n]}^{t},
$$

where $\operatorname{mean}_{\tilde{y}_{j}[n]}^{t}=\tilde{y}_{j}[n]-\sum_{p} \tilde{h}_{j, p}[n] \hat{m}_{x_{p}[n] \rightarrow \tilde{y}_{j}[n]}^{t}$ and $\operatorname{var}_{\tilde{y}_{j}[n]}^{t}=\sum_{p}\left|\tilde{h}_{j, i}\right|^{2} \hat{v}_{x_{p}[n] \rightarrow \tilde{y}_{j}[n]}^{t}+\sigma^{2}$.

Thus, the message passed from observation node $\tilde{y}_{j}[n]$ to the variable node $x_{i}[n]$ at the $t$ th iteration is approximately expressed as

$$
\mu_{\tilde{y}_{j}[n] \rightarrow x_{i}[n]}^{t}\left(x_{i}[n]\right) \propto \exp \left(-\frac{\left|\tilde{h}_{j, i} x_{i}[n]-\operatorname{mean}_{\tilde{y}_{j}[n] \rightarrow x_{i}[n]}^{t}\right|^{2}}{\operatorname{var}_{\tilde{y}_{j}[n] \rightarrow x_{i}[n]}^{t}}\right)
$$

in which the calculation of $\operatorname{mean}_{\tilde{y}_{j}[n] \rightarrow x_{i}[n]}^{t}$ and $\operatorname{var}_{\tilde{y}_{j}[n] \rightarrow x_{i}[n]}^{t}$ is simple linear operation. 
The messages passed from the bottom-most nodes are computed at the bottom-second node as follows:

$$
\begin{aligned}
& \mu_{x_{i}[n] \rightarrow \tilde{x}_{i}[l]}^{t}\left(x_{i}[n]\right)=\prod_{j} \mu_{\tilde{y}_{j}[n] \rightarrow x_{i}[n]}^{t}\left(x_{i}[n]\right) \\
& \propto \prod_{j} \exp \left(-\frac{\left|\tilde{h}_{j, i} x_{i}[n]-\operatorname{mean}_{\tilde{y}_{j}[n] \rightarrow x_{i}[n]}^{t}\right|^{2}}{\operatorname{var}_{\tilde{y}_{j}[n] \rightarrow x_{i}[n]}^{t}}\right) \\
& =\exp \left[\sum_{j}\left(-\frac{\left|\tilde{h}_{j, i} x_{i}[n]-\operatorname{mean}_{\tilde{y}_{j}[n] \rightarrow x_{i}[n]}^{t}\right|^{2}}{\operatorname{var}_{\tilde{y}_{j}[n] \rightarrow x_{i}[n]}^{t}}\right)\right] \\
& =\exp \left[\sum_{j}\left(-\frac{\left|\tilde{h}_{j, i}\right|^{2} x_{i}^{2}[n]-2 \tilde{h}_{j, i} \operatorname{mean}_{\tilde{y}_{j}[n] \rightarrow x_{i}[n]}^{t} \operatorname{var}_{\tilde{y}_{j}[n] \rightarrow x_{i}[n]}^{t}[n]+\left|\operatorname{mean}_{\tilde{y}_{j}[n] \rightarrow x_{i}[n]}^{t}\right|^{2}}{x^{2}}\right)\right] \\
& \propto \exp \left(-\frac{\left|x_{i}[n]-\xi_{x_{i}[n]}^{t}\right|^{2}}{\zeta_{x_{i}[n]}^{t}}\right) .
\end{aligned}
$$

The expression of $\xi_{x_{i}[n]}^{t}$ and $\zeta_{x_{i}[n]}^{t}$ can be obtained by the corresponding coefficients of the terms $x_{i}^{2}[n]$ and $x_{i}[n]$ which is shown as follows.

$$
\begin{aligned}
& \xi_{x_{i}[n]}^{t}=\zeta_{x_{i}[n]}^{t} \sum_{j} \frac{\tilde{h}_{j, i} \operatorname{mean}_{\tilde{y}_{j}[n] \rightarrow x_{i}[n]}^{t}}{\operatorname{var}_{\tilde{y}_{j}[n] \rightarrow x_{i}[n]}^{t}} \\
& \zeta_{x_{i}[n]}^{t}=\left(\sum_{j} \frac{\left|\tilde{h}_{j, i}\right|^{2}}{\operatorname{var}_{\tilde{y}_{j}[n] \rightarrow x_{i}[n]}^{t}}\right)^{-1} .
\end{aligned}
$$

Before the operation at the bottom-most node, the received signal is multiplied by each user's spreading sequence, but the despreading is not yet completed. Thus, after message passing from the bottom-most nodes, the following should be performed before passing upward in order to complete the despreading:

$$
\mu_{\tilde{x}_{i}[l] \rightarrow \operatorname{dec}}^{t}\left(\tilde{x}_{i}[l]\right)=\prod_{n=1+(l-1) N}^{N+(l-1) N} \mu_{x_{i}[n] \rightarrow \tilde{x}_{i}[l]}^{t}\left(x_{i}[n]\right) .
$$

The messages passed in the decoder nodes are described in $[10,23,24,26]$. Through (20) and (23), we can derive as follows:

$$
\mu_{\tilde{x}_{i}[l] \rightarrow \operatorname{dec}}^{t}\left(\tilde{x}_{i}[l]\right) \propto \prod_{k=1+(l-1) K}^{K+(l-1) K} \exp \left(-\frac{\left|x_{i}[k]-\xi_{x_{i}[k]}^{t}\right|^{2}}{\zeta_{x_{i}[k]}^{t}}\right) .
$$

In the practical operation, the messages $\mu_{x_{i}[n] \rightarrow \tilde{x}_{i}[l]}^{t}$ $\left(x_{i}[n]\right)$ are usually mapped into the log-likelihood ratios (LLRs); thus, the multiplication in (24) can be turned into summation. For the channel decoding, the message $\mu_{\tilde{x}_{i}[l] \rightarrow \operatorname{dec}}^{t}\left(\tilde{x}_{i}[l]\right)$ is also finally mapped into the LLRs of the coded symbols that are corresponding to the symbol $\tilde{x}_{i}[l]$.

$$
\mathcal{L}^{t}\left(c_{i}[l]\right)=\log \frac{\sum_{\chi_{i}^{1}} \mu_{\tilde{x}_{i}[l] \rightarrow \operatorname{dec}}^{t}\left(\tilde{x}_{i}[l]\right)}{\sum_{\chi_{i}^{0}} \mu_{\tilde{x}_{i}[l] \rightarrow \operatorname{dec}}^{t}\left(\tilde{x}_{i}[l]\right)},
$$

where $\chi_{i}^{1}$ and $\chi_{i}^{0}$ denote the subset of all the symbols with the corresponding information bit 1 and 0 , respectively. According to the LLRs, the information bits of the $i$ th user are decoded, and the extrinsic information for the next iteration $\mu_{\mathrm{dec} \rightarrow \tilde{x}_{i}[l]}^{t+1}\left(\tilde{x}_{i}[l]\right)$ is fed from the decoder, which is spreaded again yielding $\mu_{\tilde{x}_{i}[l] \rightarrow x_{i}[n]}^{t+1}\left(x_{i}[n]\right)$ to the variable nodes $x_{i}[n]$.

The BP-based algorithm can be carried out with different schedules due to the presence of cycles in the considered factor graph. The proposed algorithm adopts flooding schedule, which is suitable to be implemented in parallel. The messages are flooded from each node sets as described above, and the flooding scheme requires the least number of computations. Thus, the messages passed in the factor graph are formalized in Algorithm 1, in which in order to utilize the iterative structure of LDPC decoding, we extend the internal iterations in decoder to the global ones described above, namely one internal iteration per global iteration.

\subsection{Iterative complexity analysis of the joint iterative algorithm}

Figure 3 roughly shows the iteration settings of the proposed joint iterative algorithm and the iterative MMSE algorithm. For the joint iterative algorithm, I times global iterations are performed, the internal iterations in the LDPC decoder are incorporated into the global ones (Figure 3a), forming one global iteration. Meanwhile, for the iterative MMSE algorithm, $R$ iterations of the LDPC decoder are done during one iteration of the MMSE detection. Totally, $J$ iterations in the iterative MMSE algorithm are performed (Figure 3b). Thus, in order to achieve similar iterations for LDPC decoding, the relationship $I=$ $R \times J$ should be established.

In the following, we discuss the computation complexity per iteration about the decoding and detection, respectively.

\subsubsection{LDPC decoding}

In the proposed algorithm, the iteration of LDPC decoding is performed through the global iteration for detection. In the conventional iterative MMSE algorithm, the iteration for LDPC decoding is done separately. Both algorithms need to compute the extrinsic LLR for each code bit. Thus, the complexity of LDPC decoder per iteration per user can be regarded as the same. For fair comparison, as we discussed about Figure 3, the relationship $I=R \times J$ is established, which means the total number of the iterations for LDPC decoding are the same in both algorithms. 


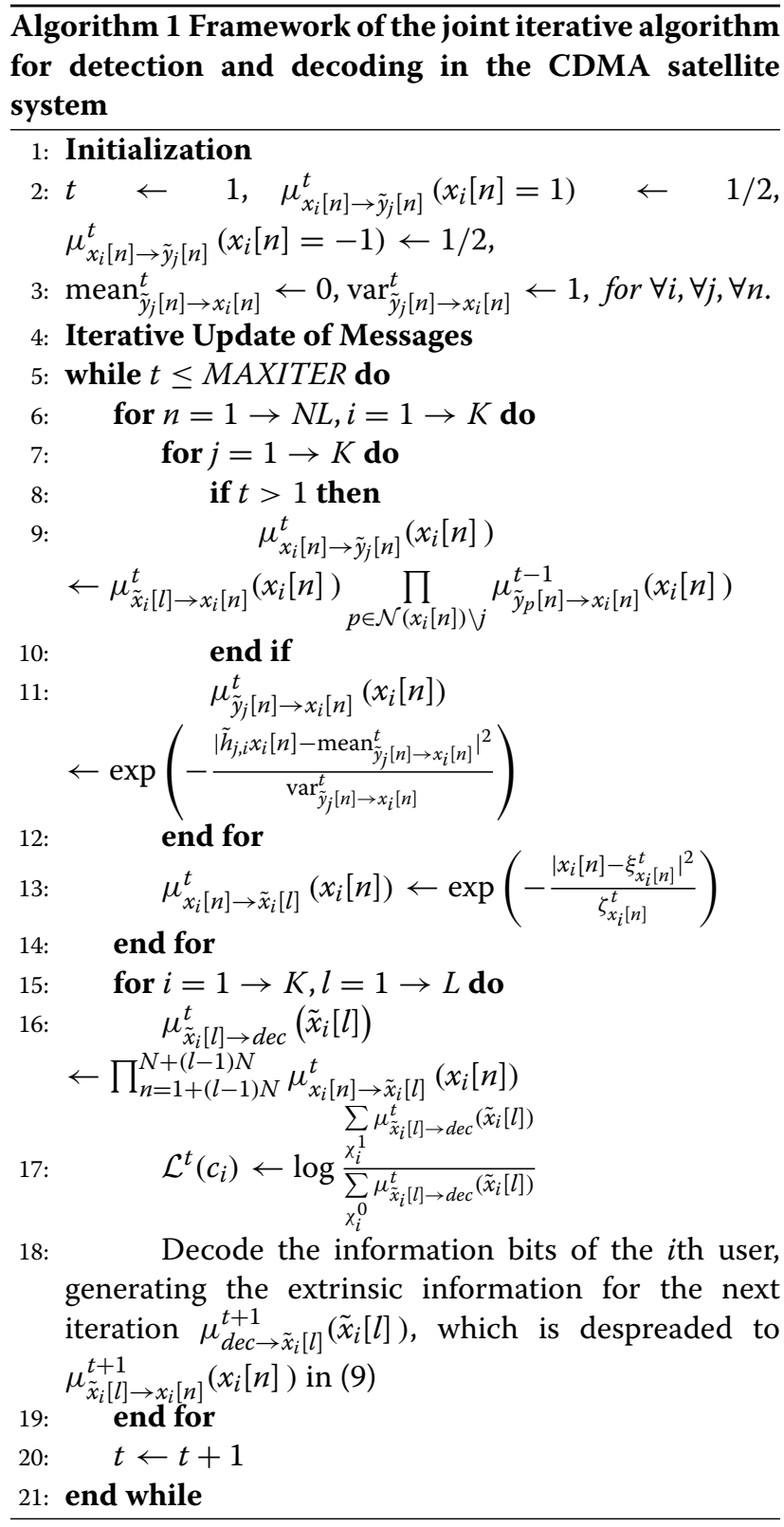

Thus, considering the iterative times, the total computation complexity of all the iterations for LDPC decoding is still the same in our following comparison.

\subsubsection{Multiuser detection}

We compare the complexity of the detection in Algorithm 1 with the iterative MMSE detection algorithm in Section II.E of [8]. Both algorithms need to compute the mean and variance in the node $x_{i}[n]$. The difference between the two algorithms is the computation of $\xi_{x_{i}[n]}^{t}$ and $\zeta_{x_{i}[n]}^{t}$, which only corresponds to the extrinsic mean and variance of $x_{i}[n]$ in the iterative MMSE detection algorithm. However, in the proposed algorithm, not only the extrinsic mean and variance but also the messages (a)

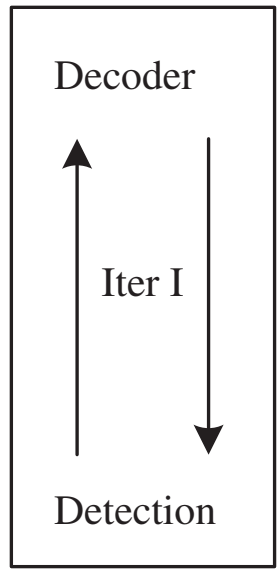

(b)

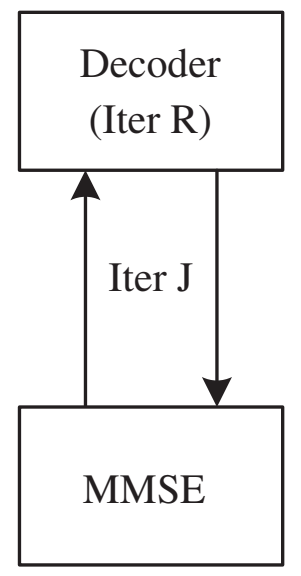

Figure 3 The sketch map of iterative structure and complexity comparison. The proposed algorithm (a) and the iterative MMSE algorithm (b)

obtained in the bottom-most nodes at the previous iteration are involved as it is stated above. The computation complexity of one iteration for multiuser detection in the proposed algorithm involves the following:

1. At the observation nodes $\tilde{y}_{j}[n]$, for $K$ users, the calculations of (19) per chip per iteration include the computation of mean $\tilde{y}_{j}[n] \rightarrow x_{i}[n]$ and $\operatorname{var}_{\tilde{y}_{j}[n] \rightarrow x_{i}[n]}^{t}$ expressed by (17) and (18), which needs $O\left(K^{2}\right)$ operations.

2. At the variable nodes $x_{i}[n]$, for $K$ users, the calculations of (20) per chip per iteration include the computation of $\xi_{x_{i}[n]}^{t}$ and $\zeta_{x_{i}[n]}^{t}$ expressed by (21) and (22), which also requires $O(K *(2 K+1))$ operations.

Thus, the computation complexity of the proposed algorithm is $O\left(K^{2}\right)$ for $K$ users per chip per iteration. Compared with the iterative MMSE algorithm in Section II-E of [8], which needs $O\left(K \times K^{3}\right)$ per chip per iteration for $K$ users, the proposed algorithm becomes more attractive in CDMA satellite systems with the number of users increasing. From the perspective of iterative times, with the similar iterative times $I=R \times J$, the total complexity of the proposed algorithm is still less than that of the conventional iterative MMSE algorithm.

\section{Simulation results}

In the simulation, we assume that the number of users is $K=6$, and the spreading factor is $N=16$. Thus, for the satellite communications on-the-move system with a middle rate (e.g., $1 \mathrm{Mbps}$ ), this means the occupied bandwidth is nearly $20 \mathrm{MHz}$, and the spectrum density is reduced by about $13 \mathrm{~dB}$. The spreading sequences are derived from gold sequences. The average performance over all users 
in the asynchronous case is considered. In the simulation, an LDPC encoder of code rate, rate $=1 / 2$, was used as the error control code. The LDPC block length was set to be $L=1,024$. We assume that the receiver knows the spreading sequences exactly and each user's propagation delay can be acquired through the timing and synchronization module. The following simulation is done in the asynchronous case.

First, we investigate the BER of the proposed algorithm at the different iterative times. For instance, the BER performances of the proposed algorithm at the 1 th, 10th, 20th, 30th, 40th, and 50th iterations are illustrated in Figure 4, respectively. The BER performance when there is only one user without mutual interferences is also shown in this figure by the curve (SU-DEC(Iter50)). It can be seen that the performance converges toward the single-user performance at higher $E_{b} / N_{0}$ with the increase of the iterative times. When the iterative times increases to more than 30 , the performance improvement is quite lower, which means the performance of the proposed algorithm becomes more stable.

We compare the performance of the proposed algorithm with that of the conventional iterative MMSE algorithm [8], in which the iterations of LDPC decoding and MMSE detection are independently performed. As the performance of the iterative LDPC decoder is verified to be quite stable after ten iterations [26], we assume the iterative times of the LDPC decoder and the MMSE detection are respectively $R=10$ and $J=2$ in the conventional iterative MMSE algorithm, which is shown by the curve (6Users-MMSE(Iter2)-DEC(Iter10)) in Figure 5. For more fair comparison, as it is discussed in Section 3.3, we assume the iterative times of the proposed algorithm $I=20$, as shown by (6UsersJoint(Iter20)). In Figure 5, the simulation results are also compared with the performance of the single-user case (SU-DEC(Iter20)) in which there are only 20 iterations of LDPC decoder and no detection. In order to compare these two algorithms more specifically, Figure 6 shows the simulation results of the case $R=10, J=5$, $I=50$, shown by the curves (6Users-MMSE(Iter5)DEC(Iter10)),(6Users-Joint(Iter50)), and the case $R=$ $25, J=2, I=50$, shown by the curves (6UsersMMSE(Iter2)-DEC(Iter25)),(6Users-Joint(Iter50)) which are also compared with the single-user performance (SUDEC(Iter50)). As we discussed in Section 3.3, in these settings about $I, J$, and $R$, the relationship $I=R \times J$ is established, which ensures that the total iterative times of decoding are the same in both algorithms. Thus, the computation complexity about LDPC decoding is the same in our comparison, and the total computation complexity of the proposed algorithm is still lower than that of the iterative MMSE algorithm, which is also discussed and compared in Section 3.3.

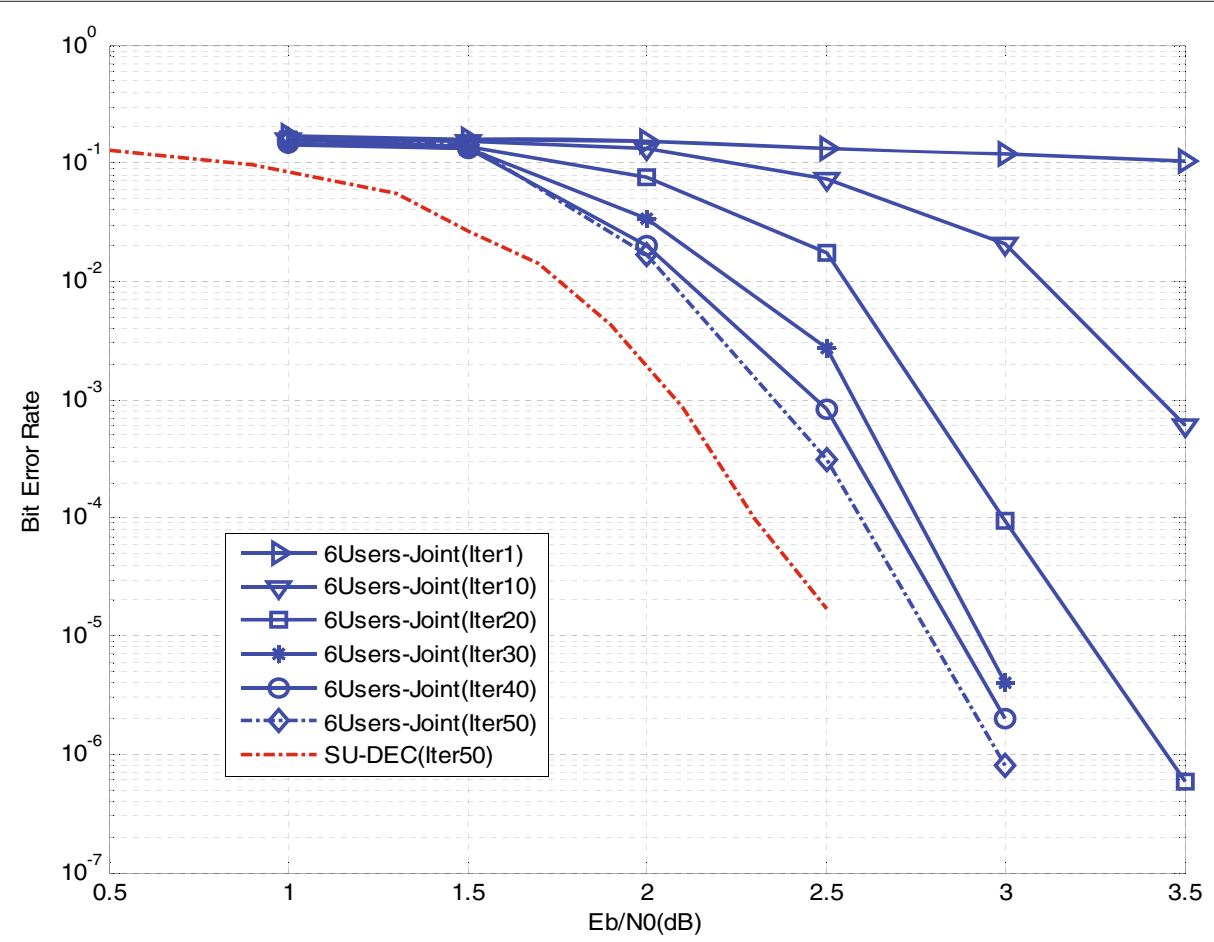

Figure 4 The BER performance of the proposed algorithm at different iterative times. The number of users is $K=6$, and the spreading factor is $N=16$. 
Figure 5 shows that after a total of 20 iterations, the performance of the proposed algorithm outperforms that of the conventional iterative MMSE algorithm by $2 \mathrm{~dB}$, and the performance is about $0.5 \mathrm{~dB}$ away from that of the single-user case. Figure 6 shows that after a total of 50 iterations, the performance of the proposed algorithm outperforms that of the conventional iterative MMSE algorithm by at least $1 \mathrm{~dB}$, and the performance is less than $0.5 \mathrm{~dB}$ away from that of the single-user case. When the iterative times are fewer, the performance improvement of the proposed algorithm is more significant. On the other hand, the curve (6Users-Joint(Iter10)) in Figure 5 shows that in order to achieve the similar performance, the proposed algorithm needs less than half of the iterative times compared with the conventional iterative MMSE algorithm.

Then, we extend the simulation to the distributed power constraints. We assume that the powers of three users are $3 \mathrm{~dB}$ higher than that of the other three users in the system. The performance of the stronger (StrongerJoint(Iter20)) and weaker (Weaker-Joint(Iter20)) users are shown in Figure 7, compared with the performance of the situation when all users have equal power (EqualJoint(Iter20)). From the results, we can see that the weaker users actually benefit from the strong interference due to the interference cancellation, whereas the stronger users suffer performance loss from the weak interference. The phenomenon was also previously observed in the iterative soft interference cancellation [5].

Next, we compare the convergences of the two algorithms. The BER performance against the number of total iterations are plotted in Figure 8. Through comparing the convergences of the two algorithms at the corresponding $E_{b} / N_{0}$, which are shown by the curves (6Users-Joint (3 dB)),(6Users-Joint (3.3 dB)) and (6UsersMMSE (3 dB)),(6Users-MMSE (3.5 dB)), it can be seen that at the same $E_{b} / N_{0}$, the BER of the proposed algorithm becomes stable after fewer iterations, which means the convergence of the proposed algorithm outperforms that of the conventional iterative MMSE algorithm. The curves (6Users-MMSE (3.8 dB)) and (6Users-MMSE (4 dB)) show that the BER performance of the iterative MMSE algorithm becomes stable at the higher $E_{b} / N_{0}$ after more iterations, and it further indicates that the convergence rate is much lower than the proposed algorithm.

As the factor graph is not sparse for CDMA system, whether the proposed BP-based joint algorithm is stable in the case of the high loading factor is investigated. Firstly, the convergence of the proposed algorithm in the case of the loading factor $K / N=5 / 8,3 / 4$, and $7 / 8$ when $N=16$ is simulated, and the results are displayed in Figure 9. It is indicated that when the loading factor is $7 / 8$, which is shown by the curves $K=14$, the performance of the proposed algorithm fails to converge. Moreover, the

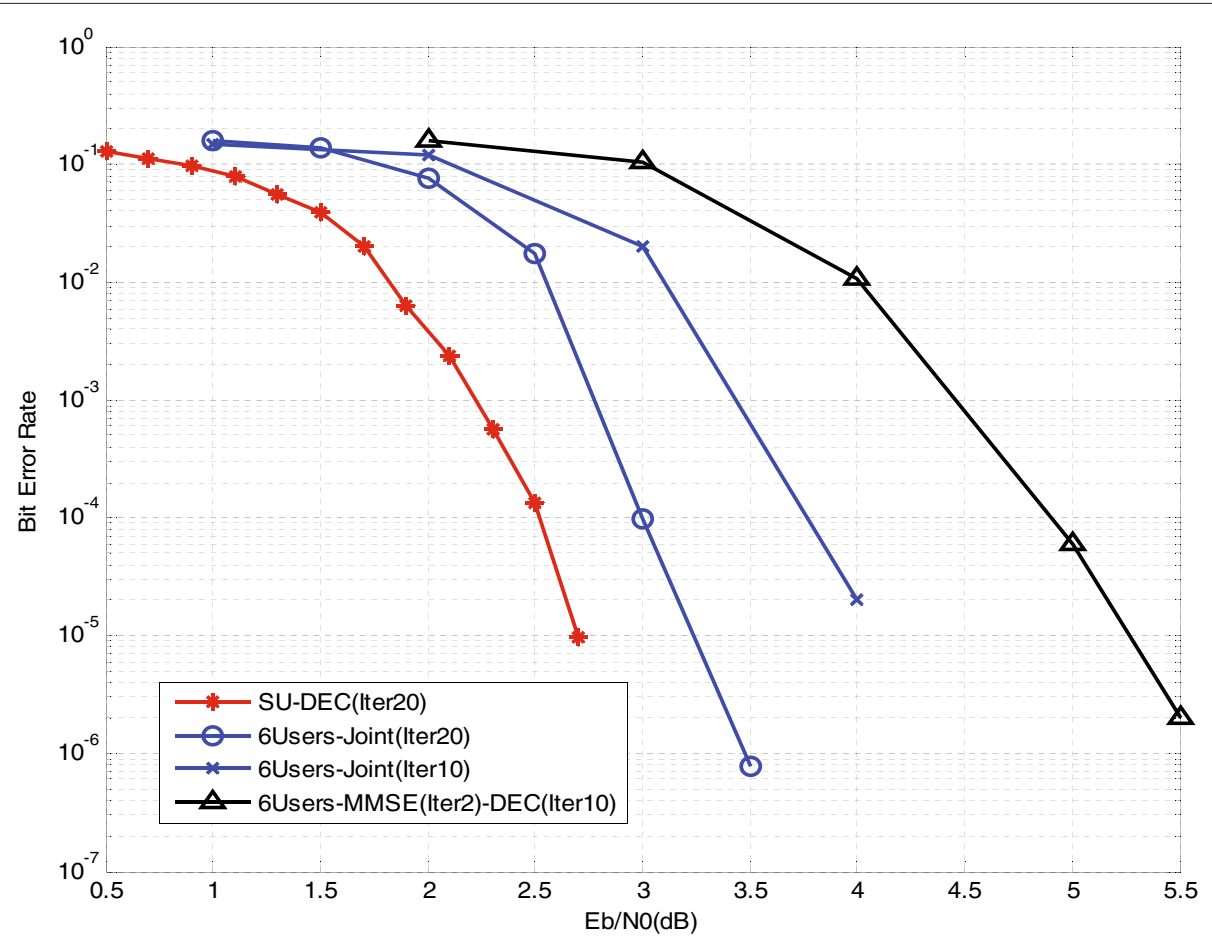

Figure 5 The BER performance of the proposed algorithm compared with that of the iterative MMSE algorithm. The number of users is $K=6$, and the spreading factor is $N=16$. The iterative times are set to be $I=20, J=2, R=10$. 


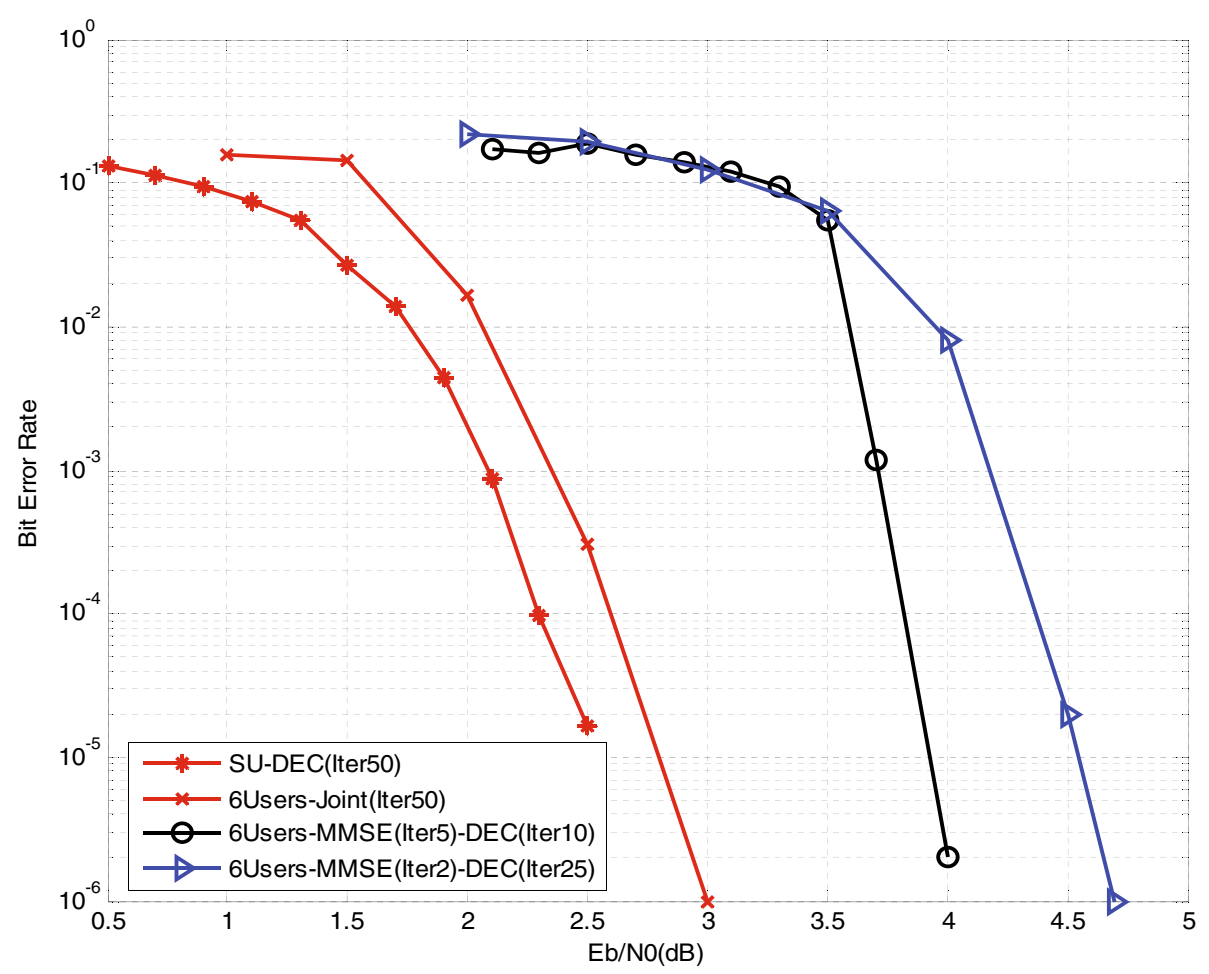

Figure 6 The BER performance of the proposed algorithm compared with that of the iterative MMSE algorithm. The number of users is $K=6$, and the spreading factor is $N=16$. The iterative times is set to be $I=50, J=5, R=10$ and $I=50, J=2, R=25$, respectively.

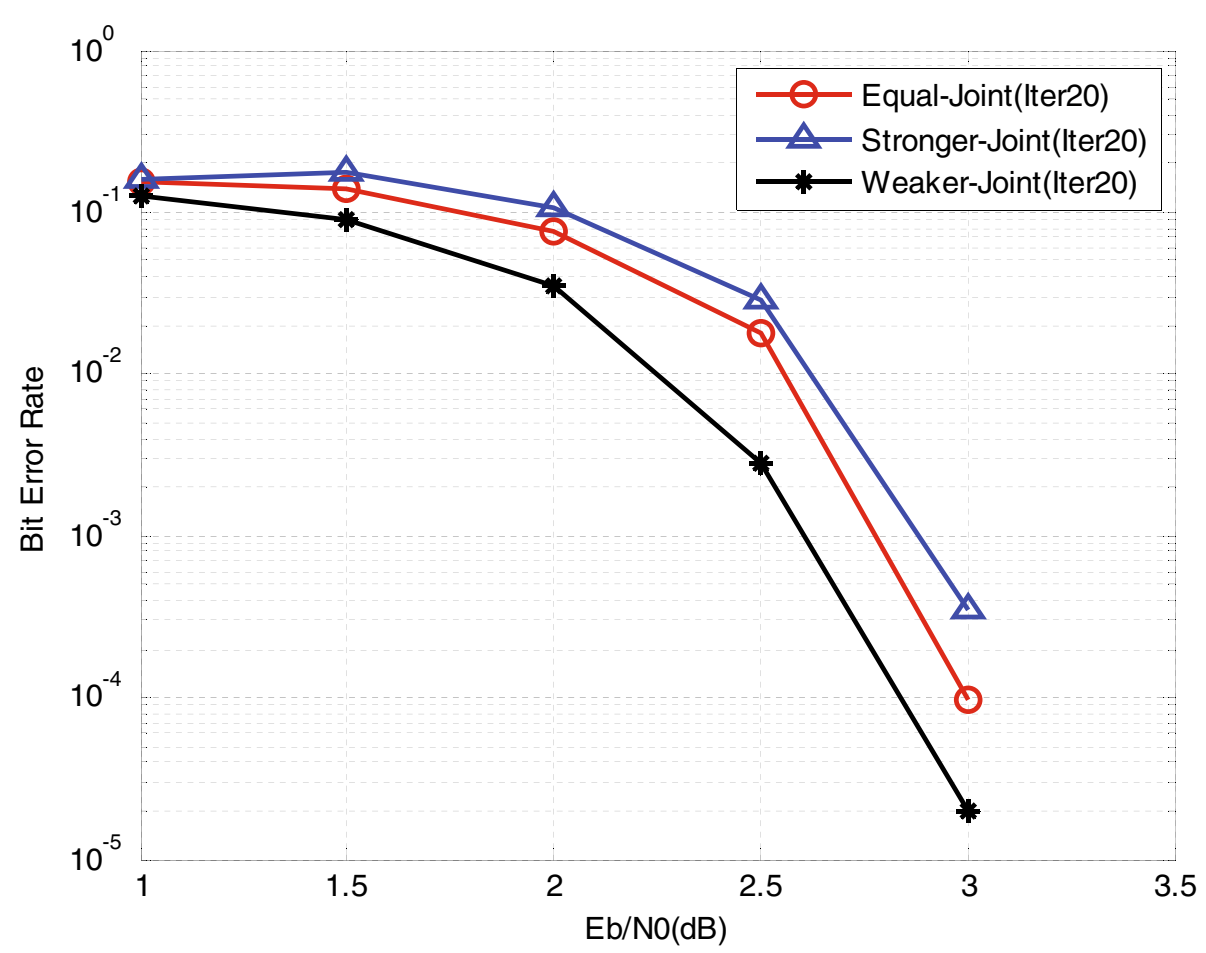

Figure 7 The BER performance of the proposed algorithm with unperfect power control. The number of users is $K=6$, and the spreading factor is $N=16$. Three users are $3 \mathrm{~dB}$ stronger than the other three. 


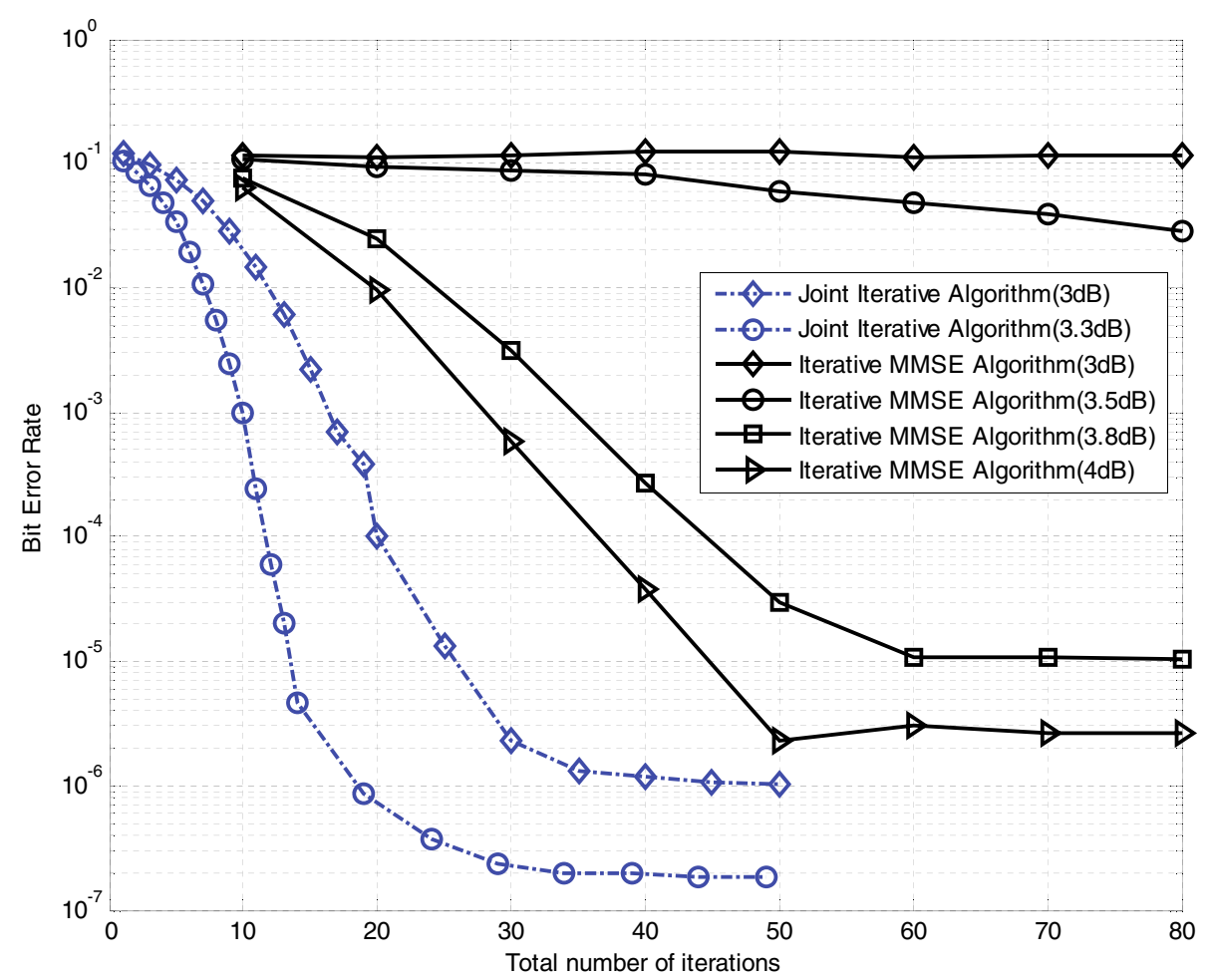

Figure 8 The convergence comparison between the proposed algorithm and the iterative MMSE algorithm. The number of users is $K=6$, and the spreading factor is $N=16$.

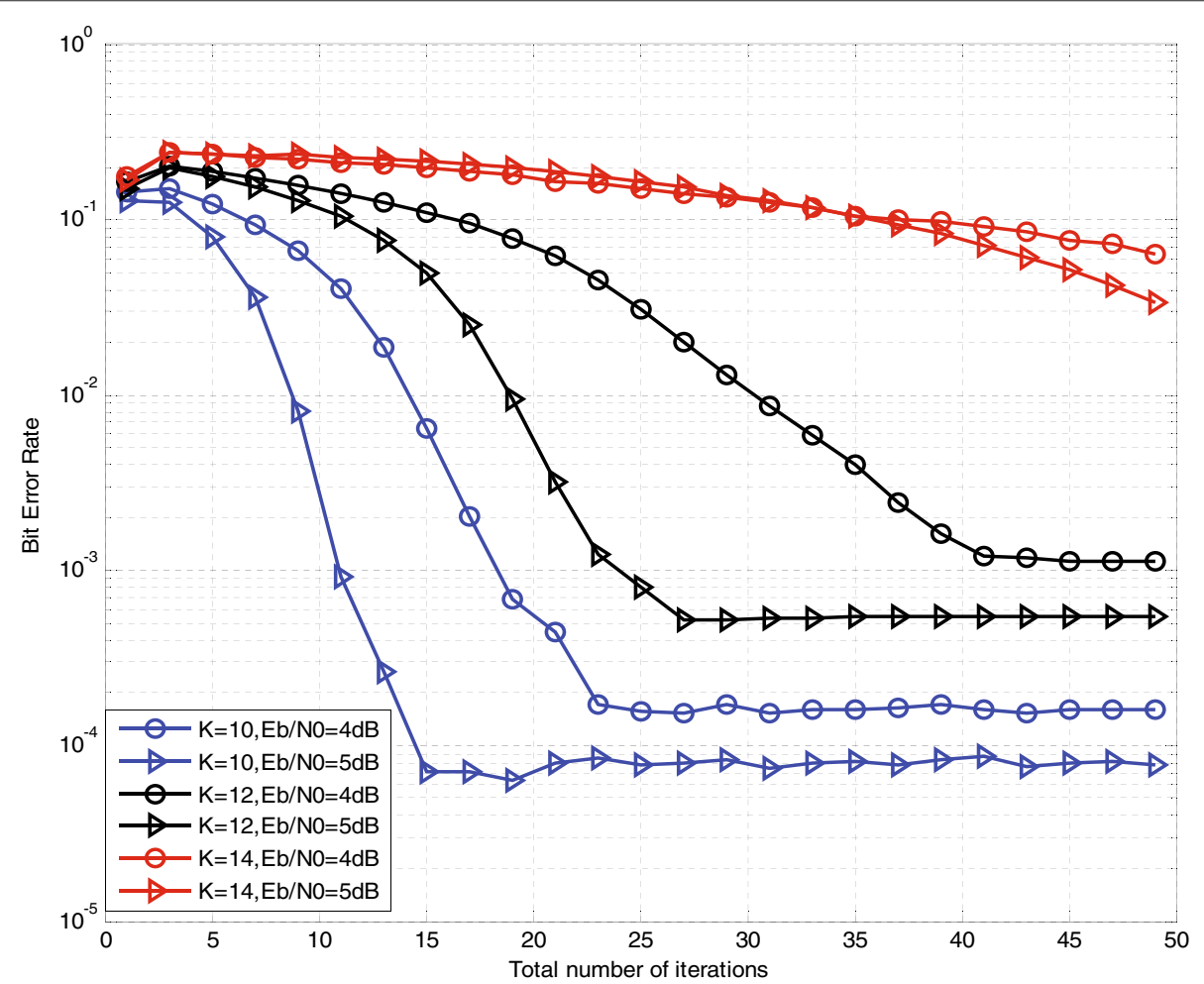

Figure 9 The convergence of the proposed joint iterative algorithm in different cases of $\boldsymbol{K} / \boldsymbol{N}$. For different number of users $k$, the spreading factor is $N=16$. 


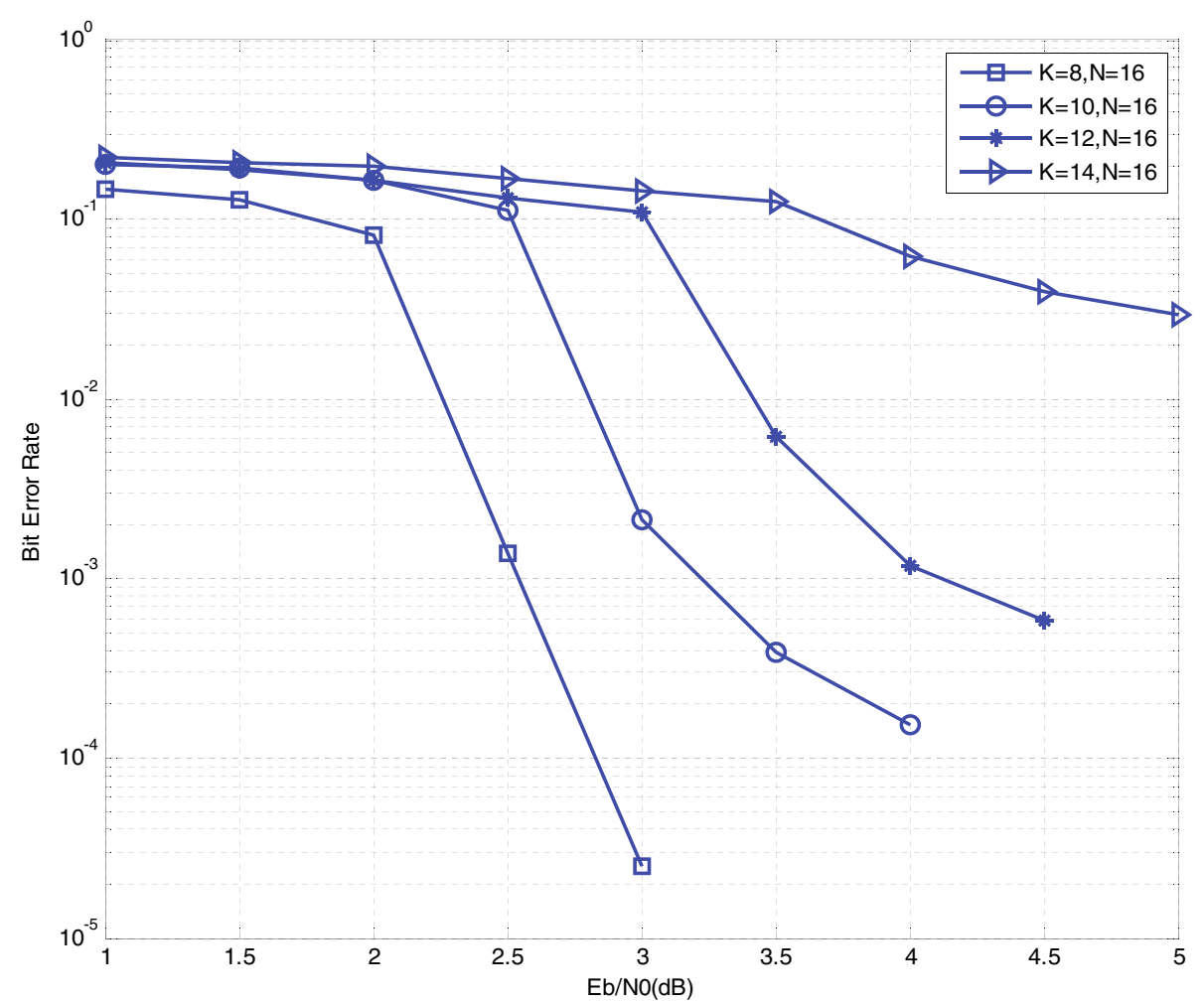

Figure 10 The BER performance of the proposed joint iterative algorithm in different cases of $K / N$. For different number of users $k$, the spreading factor is $N=16$.

BER performance of the proposed algorithm in the case of $K / N=1 / 2,5 / 8,3 / 4$, and $7 / 8$ is shown in Figure 10. It is also shown that when the loading factor is under $3 / 4$, the performance of the proposed algorithm can converge well. While in the case of the loading factor $K / N=7 / 8$ which is higher than $3 / 4$, the BER performance deteriorates. Through Figures 9 and 10, it is concluded that there is a range of the loading factor for the application of the proposed algorithm. For example, in the simulation assumption of $N=16$, when the loading factor is not higher than $3 / 4$, the proposed algorithm can converge to be stable.

\section{Conclusion}

The main contribution of this paper is the derivation of the joint chip-level iterative algorithm for CDMA multiuser detection and LDPC decoding for the asynchronous satellite communications on-the-move system in which the suppression of the MAI is essential. In particular, we formulate a factor graph over which all information bits from all users are detected and decoded through the joint iteration. In order to utilize the iterative structure of the LDPC decoder, we naturally integrate the internal iteration of the LDPC decoder and the iteration of the CDMA multiuser detection through the factor graph, becoming one global iteration. Additionally, not only the extrinsic information from the decoder but also that from the previous iteration of the detection is employed. The numerical simulations show that the performance of the proposed algorithm outperforms that of the iterative MMSE algorithm at least $1 \mathrm{~dB}$ after a total of 50 iterations and approaches the single-user performance by less than $0.5 \mathrm{~dB}$. When the iterative times are fewer, the performance gains of the proposed algorithm become more significant. On the other hand, in order to achieve the similar performance, the proposed algorithm needs less than half of the iterative times compared with the conventional iterative MMSE algorithm, which means a reduction of almost half of the joint detection and decoding latency and lower complexity for CDMA satellite systems, more suitable for engineering implementation.

\section{Competing interests}

The authors declare that they have no competing interests.

\section{Acknowledgements}

This work was supported by Co-innovation Laboratory of Aerospace Broadband Network Technology and the National Nature Science Foundation of China under grant numbers 61021001, 61132002. 


\section{Author details}

'Department of Electronic Engineering, Tsinghua University, Beijing 100084, China. ${ }^{2}$ Tsinghua Space Center, Shenzhen Key Laboratory of Wireless Broadband Communication and Signal Processing, Tsinghua University, Beijing 100084, China. ${ }^{3}$ Research Institute of Information Technology, Tsinghua University, Beijing 100084, China.

Received: 14 June 2013 Accepted: 17 September 2013

Published: 23 September 2013

\section{References}

1. VWeerackody, L Gonzalez, Mobile small aperture satellite terminals for military communications. Commun. Mag. IEEE. 45(10), 70-75 (2007)

2. M Ghotbi, M Soleymani, Multiuser detection of DS-CDMA signals using partial parallel interference cancellation in satellite communications. Selected Areas Commun. IEEE J. 22(3), 584-593 (2004)

3. S Verdú, Multiuser Detection. (Cambridge University Press, Cambridge, 1998)

4. JG Proakis, Digital Communications, 4th edn. (McGraw-Hill, New York, 2000)

5. X Wang, H Poor, Iterative (turbo) soft interference cancellation and decoding for coded CDMA. Commun. IEEE Trans. 47(7), 1046-1061 (1999)

6. H El Gamal, E Geraniotis, Iterative multiuser detection for coded CDMA signals in AWGN and fading channels. Selected Areas Commun. IEEE J. 18, 30-41 (2000)

7. M Tüchler, R Koetter, A Singer, Turbo equalization: principles and new results. Commun. IEEE Trans. 50(5), 754-767 (2002)

8. M Tüchler, A Singer, Turbo equalization: an overview. Inf. Theory, IEEE Trans. 57(2), 920-952 (2011)

9. J Pearl, Probabilistic Reasoning in Intelligent Systems: Network of Plausible Inference (Morgan Kaufmann, San Francisco, 1988)

10. H Wymeersch, Iterative Receiver Design. (Cambridge University Press, Cambridge, 2007)

11. Q Guo, L Ping, LMMSE turbo equalization based on factor graphs. Selected Areas Commun. IEEE J. 26(2), 311-319 (2008)

12. P Som, T Datta, N Srinidhi, A Chockalingam, B Rajan, Low-complexity detection in large-dimension MIMO-ISI channels using graphical models. Selected Topics Signal Process. IEEE J. 5(8), 1497-1511 (2011)

13. G Colavolpe, D Fertonani, A Piemontese, SISO detection over linear channels with linear complexity in the number of interferers. Selected Topics Signal Process. IEEE J. 5(8), 1475-1485 (2011)

14. Y Kabashima, A CDMA multiuser detection algorithm on the basis of belief propagation. J. Phys. A: Math. Gen. 36, 43 (2003)

15. A Montanari, D Tse, Analysis of belief propagation for non-linear problems: the example of CDMA (or: how to prove Tanaka's formula). IEEE Information Theory Workshop, 2006. ITW'06, Punta del Este, March 2006 (IEEE, Piscataway, 2006), pp. 160-164

16. A Montanari, D Tse, B Prabhakar, in Proceedings of the Forty-Third Allerton Conference on Communication, Control, and Computing. Belief propagation-based multi-user detection (Monticello, 28-30 September 2005)

17. D Guo, CC Wang, Multiuser detection of sparsely spread CDMA. Selected Areas Commun. IEEE J. 26(3), 421-431 (2008)

18. FJ Olivier, An LDPC error control strategy for low earth orbit satellite communication link applications. MScEng thesis, University of Stellenbosch, 2009

19. Y Pei, C Liu, H Feng, Y Shi, An LDPC-based physical layer solution in satellite interactive system. Commun. Comput. Inf. Sci. 163, 425-430 (2011)

20. V Weerackody, L Gonzalez, Performance of satellite communications on the move systems in the presence of antenna pointing errors. IEEE Military Communications Conference, 2006, Washington, DC, October 2006 (IEEE, Piscataway, 2006), pp. 1-7

21. F Kschischang, B Frey, HA Loeliger, Factor graphs and the sum-product algorithm. Inf. Theory IEEE Trans. 47(2), 498-519 (2001)

22. J Loeliger, HA Dauwels, J Hu, S Korl, L Ping, F Kschischang, The factor graph approach to model-based signal processing. Proc. IEEE. 95(6), 1295-1322 (2007)

23. R McEliece, D MacKay, JF Cheng, Turbo decoding as an instance of Pearl's "belief propagation" algorithm. Selected Areas Commun. IEEE J. 16(2), 140-152 (1998)
24. DJC MacKay, Good error-correcting codes based on very sparse matrices, IEEE Trans. Inf. Theory. 45(2), 399-431 (2003)

25. CM Bishop, Pattern Recognition and Machine Learning (Springer, New York, 2006)

26. J Chen, A Dholakia, E Eleftheriou, M Fossorier, XY Hu, Reduced-complexity decoding of LDPC codes. Commun. IEEE Trans. 53(8), 1288-1299 (2005)

doi:10.1186/1687-1499-2013-234

Cite this article as: Gu et al:: Belief propagation-based joint iterative algorithm for detection and decoding in asynchronous CDMA satellite systems. EURASIP Journal on Wireless Communications and Networking 2013 2013:234.

\section{Submit your manuscript to a SpringerOpen ${ }^{\mathcal{O}}$ journal and benefit from:}

- Convenient online submission

Rigorous peer review

- Immediate publication on acceptance

- Open access: articles freely available online

- High visibility within the field

- Retaining the copyright to your article

Submit your next manuscript at $\gg$ springeropen.com 